\title{
On the Construction of High Dimensional Simple Games
}

\author{
Martin Olsen \\ BTECH, Aarhus University \\ Denmark \\ martino@btech.au.dk
}

\author{
Sascha Kurz \\ University of Bayreuth \\ Germany \\ sascha.kurz@uni-bayreuth.de
}

\author{
Xavier Molinero* \\ Universitat Politècnica de Catalunya \\ Spain \\ xavier.molinero@upc.edu
}

\begin{abstract}
Every simple game can be written as the intersection of a finite number of weighted games. The smallest possible such number is the dimension of a simple game. Taylor and Zwicker have constructed simple games with $n$ players and dimension at least $2^{\frac{n}{2}-1}$. By using theory on error correcting codes, we construct simple games with dimension $2^{n-o(n)}$. Moreover, we show that there are no simple games with dimension $n$ times higher than our games. Our results hold for all $n$.
\end{abstract}

Keywords: simple games, weighted games, dimension, coding theory, Hamming distance

MSC: 91B12, 91A12, 68P30

\section{Introduction}

Simple games [12] are models of voting systems without abstention where voters vote either "yes" or "no" when they are presented to a proposal. For a simple game we simply specify the coalitions of voters for which a proposal passes if the voters in the coalition are the "yes"-voters.

Some real world voting systems are constructed using several sets of weights with a weight assigned to every voter for each set. A coalition of voters can make a proposal pass if the sum of the weights of the voters in every set of weights meets or exceeds a quota defined for that set. Actually, any voting system can be implemented in this way

\footnotetext{
${ }^{*}$ Research partially funded by Grant MTM 2012-34426 from the Spanish Economy and Competitiveness Ministry.
} 
no matter how it is defined [12] and the dimension is defined as the minimum number of weight sets needed to implement a voting system in this way. Unicameral systems have dimension 1 , bicameral systems have dimension 2 and there are also real world voting systems with dimension 3 or higher $[2,4,8]$.

\subsection{Contribution}

Taylor and Zwicker [12] have constructed simple games with dimension at least $2^{\frac{n}{2}-1}$ where $n$ is the number of voters. Using theory on error correcting codes, we construct voting systems with a dimension provably much closer to a theoretical upper bound. To be a little more specific, we significantly improve the $2^{\frac{n}{2}-1}$ lower bound to $2^{n-o(n)}$ for any $n$. The dimensions of our games are within a factor $n$ away from the theoretical upper bound $\left(\begin{array}{c}n \\ \lfloor n / 2\rfloor\end{array}\right)$ for any $n$. Our construction can also be used to produce simple games with high dimension involving only a few players.

\subsection{Related Work}

In [3] the construction of a simple game with $n$ players and a dimension of at least $\left(\begin{array}{c}n \\ n / 2\end{array}\right) / 2$ was claimed for all $n \equiv 2(\bmod 4)$. We will see later that this result is flawed. Essentially, Elkind et al. [3] consider the same game as Taylor and Zwicker but they analyze the dimension from another point of view. We will perform a more detailed comparison with the work of Taylor and Zwicker and Elkind et al. in Section 3 where we introduce the simple games forming the basis of our results.

Freixas and Puente [5] show how to construct games with high dimension for other values of $n$ using the composition of unanimity games without improving the $2^{\frac{n}{2}-1}$ bound given by Taylor and Zwicker [12]. The dimensions of real world voting systems are also analyzed in several papers $[2,4,7,8,11]$.

\subsection{Outline}

In Section 2 we introduce some notation and formally define the considered concepts in the paper. We also state a well known theoretical upper bound for the dimension for later use in this section. The games that form the basis for our results are introduced in Section 3. Finally, Section 4 contains the proofs of high dimension and a theorem that forms the main contribution of the paper.

\section{Preliminaries}

A simple game $\Gamma(N, W)$ is a pair where $N=\{1, \ldots, n\}$ for some positive integer $n$ and $W$ is a collection of subsets of $N$ such that the following conditions are met: $N \in W, \emptyset \notin W$, and $S \in W \wedge S \subseteq R \subseteq N \Rightarrow R \in W$. A coalition is a subset of $N$ and the members of $N$ are referred to as players. A coalition is winning if it is a member of $W$ and it is said to be losing otherwise. A simple game $\Gamma(N, W)$ is weighted if there exists a quota $q \in \mathbb{R}$ and weights $w_{1}, w_{2}, \ldots, w_{n} \in \mathbb{R}$ such that 
$S \in W$ if and only if $\sum_{i \in S} w_{i} \geq q$. The intersection $\Gamma_{1}\left(N, W_{1}\right) \cap \Gamma_{2}\left(N, W_{2}\right)$ of two simple games $\Gamma_{1}\left(N, W_{1}\right)$ and $\Gamma_{2}\left(N, W_{2}\right)$ is the simple game $\Gamma\left(N, W_{1} \cap W_{2}\right)$.

A losing coalition is a maximal losing coalition if any proper superset of the coalition is winning. The family consisting of all maximal losing coalitions is denoted by $L^{M}$. Taylor and Zwicker [12] have shown that any simple game can be written as the intersection of $\left|L^{M}\right|$ weighted games $\Gamma_{T}, T \in L^{M}$, where a coalition $S$ wins in $\Gamma_{T}$ if $S \cap(N \backslash T) \neq \emptyset$. A player in $N \backslash T$ has weight 1 and all other players have weight 0 in the game $\Gamma_{T}$ that has quota 1.

The dimension $d$ of a simple game $\Gamma$ is the smallest positive integer such that $\Gamma=$ $\cap_{i=1}^{d} \Gamma_{d}$ where the games $\Gamma_{i}, i \in\{1,2, \ldots, d\}$, are weighted. It is not possible to find a maximal losing coalition that is contained in another maximal losing coalition so using Sperners Lemma [9] we conclude that $\left|L^{M}\right| \leq\left(\begin{array}{c}n \\ \left\lfloor\frac{n}{2}\right\rfloor\end{array}\right)$. From the construction of Taylor and Zwicker we obtain the following theoretical upper bound:

$$
d \leq\left|L^{M}\right| \leq \min \left(2^{n}-|W|,\left(\begin{array}{c}
n \\
\left\lfloor\frac{n}{2}\right\rfloor
\end{array}\right)\right) .
$$

To give an impression of how this upper bound relates to $2^{n}$ we can use the the following double inequality that holds for all even positive integers $n$ [10]:

$$
\sqrt{\frac{2}{\pi n}}\left(1-\frac{1}{4 n}\right) 2^{n} \leq\left(\begin{array}{l}
n \\
\frac{n}{2}
\end{array}\right) \leq \sqrt{\frac{2}{\pi n}}\left(1-\frac{2}{9 n}\right) 2^{n} .
$$

For odd $n$, we can use $\left(\begin{array}{c}n \\ \left\lfloor\frac{n}{2}\right\rfloor\end{array}\right)=\left(\begin{array}{c}n-1 \\ \frac{n-1}{2}\end{array}\right) \frac{2 n}{n+1}$ and obtain the following inequalities:

$$
\begin{aligned}
& \left(\begin{array}{c}
n \\
\left\lfloor\frac{n}{2}\right\rfloor
\end{array}\right) \geq \frac{n}{n+1} \sqrt{\frac{2}{\pi(n-1)}}\left(1-\frac{1}{4(n-1)}\right) 2^{n}, \\
& \left(\begin{array}{c}
n \\
\left\lfloor\frac{n}{2}\right\rfloor
\end{array}\right) \leq \frac{n}{n+1} \sqrt{\frac{2}{\pi(n-1)}}\left(1-\frac{2}{9(n-1)}\right) 2^{n} .
\end{aligned}
$$

For a bit vector $x=x_{1} x_{2} \ldots x_{n} \in\{0,1\}^{n}$ with $n$ bits we let $S_{x}$ be the coalition where $i \in S$ if and only $x_{i}=1$. For a coalition $S \subseteq N$ we define the bit vector $x_{S}$ accordingly. We use the notation $\bar{x}$ and $\bar{S}$ for complements for bit vectors and sets respectively. The Hamming weight $h w(x)$ of a bit vector $x$ is the number of 1bits in $x$ : $h w(x)=\left|\left\{i: x_{i}=1\right\}\right|$. The Hamming distance $d(x, y)$ between two bit vectors $x$ and $y$ is the number of bit positions where the bits in $x$ and $y$ are different: $d(x, y)=\left|\left\{i: x_{i} \neq y_{i}\right\}\right|$.

\section{The Construction}

In this section, we present a generic recipe for constructing the simple games forming the basis for our results. Throughout the paper, we let $\mathcal{C} \subseteq\{0,1\}^{n}$ denote a set of bit vectors of length $n$ having positive Hamming weight satisfying this condition:

$$
\forall x \neq y \in \mathcal{C}:|h w(x)-h w(y)|<d(x, y)-2
$$


For $x \in \mathcal{C}$ we define the simple game $\Gamma_{x}$ with players $N$ as follows: $S$ wins in $\Gamma_{x}$ if and only if $S \cap S_{x} \neq \emptyset$. The simple game $\Gamma_{\mathcal{C}}$ is now defined by $\Gamma_{\mathcal{C}}=\cap_{x \in \mathcal{C}} \Gamma_{x}$. In other words, a set $S$ is winning if and only if $S$ is a so-called hitting set for the collection of sets $\left\{S_{x}\right\}_{x \in \mathcal{C}}$.

Example 1. Let $\mathcal{C}$ be defined as follows for $n=8$ :

$$
\mathcal{C}=\{00001111,11000000,00111100\}
$$

The Hamming weights of the vectors 00001111 and 11000000 differ by 2 but their Hamming distance is 6 . So (5) holds for these vectors. Coalition $\{1,5\}$ is winning in $\Gamma_{\mathcal{C}}$ since it intersects the sets $\{5,6,7,8\},\{1,2\}$ and $\{3,4,5,6\}$. The bit vector 10001000 that corresponds to the set $\{1,5\}$ shares at least one 1 -bit with all members of $\mathcal{C}$.

\subsection{Comparison with Other Games}

As promised in the introduction, we will relate the games $\Gamma_{\mathcal{C}}$ to the game used by Taylor and Zwicker [12, Theorem 1.7.5]. For an odd integer $k$, Taylor and Zwicker let $N=N_{1} \cup N_{2}$ for two disjoint sets $N_{1}$ and $N_{2}$ with $\left|N_{1}\right|=\left|N_{2}\right|=k$ and a coalition $S$ wins if 1) $|S|>k$, or 2) $|S|=k$ with $\left|S \cap N_{1}\right|$ even. The complements of the maximal losing coalitions are the sets satisfying 2). Thus, using the terms of this paper, Taylor and Zwicker use a game $\Gamma_{\mathcal{C}}$ where $\mathcal{C}$ contains bit vectors with Hamming weight $\frac{n}{2}$ and Hamming distance at least 2. Taylor and Zwicker use a subset of $\mathcal{C}$ with cardinality $2^{\frac{n}{2}-1}$ satisfying (5) as an ingredient in the proof of their $2^{\frac{n}{2}-1}$ lower bound.

It should be stressed that it requires careful analysis to see this implicit connection to the work by Taylor and Zwicker. We use a more generic approach allowing an improvement of the lower bound to $2^{n-o(n)}$ where we explicitly define a coalition to be winning if it intersects any member in a family of sets with a structure inspired by the structure of error correcting codes. Another thing to note is that coalitions with fewer than $\frac{n}{2}$ members can win in games defined by our approach as illustrated by Example 1.

Elkind et al. [3, Theorem 8] claim to have produced an example of a simple game on $n=2 k$ players with a dimension of at least $\left(\begin{array}{c}2 k \\ k\end{array}\right) / 2$, where $k$ is an arbitrary odd integer but - as we will see below - the proof is flawed. The losing coalitions are those where the corresponding bit vector either has a Hamming weight of at most $k-1$ or a Hamming weight of exactly $k$ and a Hamming distance to $1^{k} 0^{k}$ that is divisible by 4 . Swapping the first $k$ and the last $k$ players gives a simple game that coincides with that of [12]. It means that the example given by Elkind et al. [3, Theorem 8] has dimension at least $2^{k-1}$.

Moreover, the flaw ${ }^{1}$ of the corresponding proof happens where it is saying that if $x$ is the bit vector of a losing coalition and $x_{i} \neq x_{j}$, then switching $x_{i}$ and $x_{j}$ results in a bit vector of a winning coalition. An explicit counter example for $n=6$ is given by the characteristic vectors 100110 and 010110 which both represent losing coalitions.

\footnotetext{
${ }^{1}$ We would like to thank Edith Elkind for directly pointing to the position where the proof breaks down in a private communication.
} 


\subsection{A Dimension Lemma}

We now prove a lemma explicitly stating the dimension of our games.

Lemma 1. The dimension of $\Gamma_{\mathcal{C}}$ is $|\mathcal{C}|$.

Proof. The game $\Gamma_{x}, x \in \mathcal{C}$, is clearly weighted so the dimension of $\Gamma_{\mathcal{C}}$ is not higher than $|\mathcal{C}|$.

We now assume that the dimension of $\Gamma_{\mathcal{C}}$ is less than $|\mathcal{C}|$. Let $L_{x}=N \backslash S_{x}$ for $x \in \mathcal{C}$. The coalition $L_{x}$ is clearly a losing coalition in $\Gamma_{\mathcal{C}}$ because $L_{x} \cap S_{x}=\emptyset$. Using the pigeonhole principle, we conclude that there are $x, y \in \mathcal{C}$ with $x \neq y$ such that $L_{x}$ and $L_{y}$ lose in the same weighted game $\Gamma^{\prime}$ where $\Gamma^{\prime}$ is one of the less than $|\mathcal{C}|$ weighted games whose intersection is $\Gamma_{\mathcal{C}}$.

By considering basic properties for the Hamming distance and the Hamming weight we observe that (5) also holds if we replace $x$ and $y$ with their complements $\bar{x}$ and $\bar{y}$. If one of the vectors $\bar{x}$ or $\bar{y}$ had all 1-bits in the $d(\bar{x}, \bar{y})$ positions where the two vectors differ then the left hand side of (5) would be $d(\bar{x}, \bar{y})$ and (5) would not hold. We therefore conclude that there are players $p_{x} \in L_{x} \backslash L_{y}$ and $p_{y} \in L_{y} \backslash L_{x}$. We let $A$ and $B$ be the coalitions obtained if $L_{x}$ and $L_{y}$ swap these players: $A=\left(L_{x} \backslash\left\{p_{x}\right\}\right) \cup\left\{p_{y}\right\}$ and $B=\left(L_{y} \backslash\left\{p_{y}\right\}\right) \cup\left\{p_{x}\right\}$.

We now show that $A$ and $B$ are winning coalitions in $\Gamma_{\mathcal{C}}$. Without loss of generality, we consider the coalition $A$. It is clear that $x_{A}$ and $x$ share a 1-bit so $A$ wins in $\Gamma_{x}$. Now let us assume that there is a member $z$ of $\mathcal{C} \backslash\{x\}$ such that $A$ loses in $\Gamma_{z}$. In other words, $x_{A}$ and $z$ do not share a 1-bit. The vector $x_{A}$ is obtained by flipping a 0-bit and a 1-bit in the vector $\bar{x}$ :

$$
d\left(x_{A}, \bar{z}\right) \geq d(\bar{x}, \bar{z})-2 .
$$

The $d\left(x_{A}, \bar{z}\right)$ bits shared by $x_{A}$ and $z$ are all 0 in which case we have the following:

$$
d\left(x_{A}, \bar{z}\right)+h w\left(x_{A}\right)+h w(z)=n .
$$

We now use $h w\left(x_{A}\right)=n-h w(x)$ together with (7):

$$
d\left(x_{A}, \bar{z}\right)=h w(x)-h w(z) .
$$

By using $d(x, z)=d(\bar{x}, \bar{z})$ and (6) and (8) we obtain the following inequality:

$$
h w(x)-h w(z) \geq d(x, z)-2 .
$$

Since (9) contradicts (5), we conclude that $A$ wins in $\Gamma_{z}$ for any $z \in \mathcal{C}$. Consequently, $A$ also wins in $\Gamma_{\mathcal{C}}$.

Summing up, we now have two coalitions $L_{x}$ and $L_{y}$ that lose in $\Gamma^{\prime}$ and we can obtain two winning coalitions in $\Gamma_{\mathcal{C}}$ if $L_{x}$ and $L_{y}$ swap two players. These coalitions also win in $\Gamma^{\prime}$ and we obtain a contradiction since this would mean that the total weight in $\Gamma^{\prime}$ of the players in $L_{x}$ and $L_{y}$ has increased.

It is worth noting that the dimension of the game $\Gamma_{\mathcal{C}}$ is $\left|L^{M}\right|$ since $L^{M}=\left\{L_{x}\right\}_{x \in \mathcal{C}}$.

If we can construct games with dimension $m$ using our approach we can also construct games with dimension $m^{\prime}$ for every $m^{\prime} \leq m$ as expressed by the following corollary: 
Corollary 1. Let $\Gamma_{\mathcal{C}}$ be a simple game with $n$ players and dimension $m$, then there are simple games with $n$ players and dimension $m^{\prime}, 1 \leq m^{\prime} \leq m$.

Proof. Just delete some elements from $\mathcal{C}$.

\section{Proof of High Dimension}

The key question we will deal with in this section is the following: Can we find families $\mathcal{C}$ of bit vectors with high cardinality satisfying (5)? According to Lemma 1, this would automatically give us games with high dimension. From the theory on error correcting codes we know how to construct relatively large families of bit vectors where no two bit vectors have a Hamming distance less than 4 . These families are the so called single error correcting and double error detecting codes - SECDED codes. If we pick such a code where all vectors have the same Hamming weight - a constant weight code - we clearly have a family $\mathcal{C}$ satisfying (5). This observation is the basis for the proofs in this section.

It is important to stress that constant weight SECDED codes are not the only families satisfying the generic recipe (5). There are many other families that satisfy (5) but we will use constant weight SECDED codes to construct our games with high dimension. In other words, there might be families with larger cardinalities compared to constant weight SECDED codes satisfying (5).

Agrell et al. [1] present lower bounds for cardinalities of constant weight SECDED codes. These lower bounds can be directly translated to lower bounds for dimensions for simple games if we use Lemma 1. This allows us to set up Table 1 that compares the dimensions of the games produced using composition of unanimity games [5] with the dimensions of the games based on our approach and the lower bounds from [1]. The first column displays $n$. The second column presents the dimensions of the games from [5] and [12]. The third column contains the dimensions of the games produced using our approach and constant weight SECDED codes. Finally, the last column shows the upper bound $\left(\begin{array}{c}n \\ \lfloor n / 2\rfloor\end{array}\right)$.

We are now ready to look all other values of $n$. Initially, we consider the case where $n$ is a power of 2 .

Lemma 2. Let $n=2^{m}$ where $m$ is an integer, $m \geq 3$. There is a set of bit vectors $\mathcal{C} \subseteq\{0,1\}^{n}$ satisfying (5) with

$$
|\mathcal{C}|=\frac{2}{n}\left(\frac{1}{2}\left(\begin{array}{l}
n \\
\frac{n}{2}
\end{array}\right)+(n-1)\left(\begin{array}{c}
\frac{n}{2}-1 \\
\frac{n}{4}
\end{array}\right)\right) .
$$

Proof. Let $t=2^{m}-1$. The enumerator polynomial for an error correcting code is a polynomial where the $i$ 'th coefficient, $a_{i}$, is the number of bit vectors of Hamming weight $i$. According to [13], the enumerator polynomial for the well known Hamming $[t, t-m]$ code that contains bit vectors of length $t$ is:

$$
A(x)=\frac{(1+x)^{t}+t(1-x)\left(1-x^{2}\right)^{(t-1) / 2}}{t+1} .
$$


Table 1: A comparison of the dimensions of the games produced using composition of unanimity games and the dimensions of the games based on our approach.

\begin{tabular}{|r|c|c|c|}
\hline$n$ & Unanimity games & Our approach & $\left(\begin{array}{c}n \\
\lfloor n / 2\rfloor\end{array}\right)$ \\
\hline 6 & 4 & 4 & 20 \\
7 & 4 & 7 & 35 \\
8 & 8 & 14 & 70 \\
9 & 9 & 18 & 126 \\
10 & 16 & 36 & 252 \\
11 & 18 & 66 & 462 \\
12 & 32 & 132 & 924 \\
13 & 36 & 166 & 1716 \\
14 & 64 & 325 & 3432 \\
15 & 81 & 585 & 6435 \\
16 & 128 & 1170 & 12870 \\
17 & 162 & 1770 & 24310 \\
18 & 256 & 3540 & 48620 \\
19 & 324 & 6726 & 92378 \\
20 & 512 & 13452 & 184756 \\
\hline
\end{tabular}

Let $i=\frac{t-1}{2}\left(i=2^{m-1}-1\right.$ is odd and $i+1$ is even $)$ :

$$
\begin{gathered}
a_{i}=\frac{1}{t+1}\left(\left(\begin{array}{c}
t \\
i
\end{array}\right)+t(-1)^{\frac{i+1}{2}}\left(\begin{array}{c}
i \\
\frac{i-1}{2}
\end{array}\right)\right) \\
a_{i+1}=\frac{1}{t+1}\left(\left(\begin{array}{c}
t \\
i+1
\end{array}\right)+t(-1)^{\frac{i+1}{2}}\left(\begin{array}{c}
i \\
\frac{i+1}{2}
\end{array}\right)\right)=a_{i}
\end{gathered}
$$

The extended code Hamming $[t+1, t-m]$ is a SECDED code. We can now let $\mathcal{C}$ be the subset of the extended code containing the bit vectors with Hamming weight $\frac{n}{2}$. This is a constant weight SECDED code satisfying (5).

Set $n=t+1=2 i+2$. The number of bit vectors in the extended code with Hamming weight $\frac{n}{2}=i+1$ is $a_{i}+a_{i+1}=2 a_{i+1}$ :

$$
2 a_{i+1}=\frac{2}{n}\left(\left(\begin{array}{c}
n-1 \\
\frac{n}{2}
\end{array}\right)+(n-1)(-1)^{\frac{n}{4}}\left(\begin{array}{c}
\frac{n}{2}-1 \\
\frac{n}{4}
\end{array}\right)\right)
$$

For $n \geq 8$ we have:

$$
2 a_{i+1}=\frac{2}{n}\left(\left(\begin{array}{c}
n-1 \\
\frac{n}{2}
\end{array}\right)+(n-1)\left(\begin{array}{c}
\frac{n}{2}-1 \\
\frac{n}{4}
\end{array}\right)\right)
$$

We now use

$$
\left(\begin{array}{l}
n \\
\frac{n}{2}
\end{array}\right)=\left(\begin{array}{c}
n-1 \\
\frac{n}{2}
\end{array}\right)+\left(\begin{array}{c}
n-1 \\
\frac{n}{2}-1
\end{array}\right)=2\left(\begin{array}{c}
n-1 \\
\frac{n}{2}
\end{array}\right)
$$


to obtain

$$
2 a_{i+1}=\frac{2}{n}\left(\frac{1}{2}\left(\begin{array}{l}
n \\
\frac{n}{2}
\end{array}\right)+(n-1)\left(\begin{array}{c}
\frac{n}{2}-1 \\
\frac{n}{4}
\end{array}\right)\right) .
$$

We now state our main theorem where we also consider values of $n$ that are not powers of 2 .

Theorem 1. For any positive integer $n$ there is a simple game with $n$ players and dimension d satisfying:

$$
d \geq \frac{1}{n}\left(\begin{array}{c}
n \\
\left\lfloor\frac{n}{2}\right\rfloor
\end{array}\right) .
$$

If $n=2^{m}$ for an integer $m \geq 3$ then there is a simple game with $n$ players and dimension $d$ such that

$$
d=\frac{1}{n}\left(\begin{array}{c}
n \\
\frac{n}{2}
\end{array}\right)+\frac{2(n-1)}{n}\left(\begin{array}{c}
\frac{n}{2}-1 \\
\frac{n}{4}
\end{array}\right) .
$$

Proof. Graham and Sloane [6] have shown that there is constant weight SECDED code with Hamming weight $w$ with cardinality at least $\frac{1}{n}\left(\begin{array}{l}n \\ w\end{array}\right)$ for any $w$. For $w=\left\lfloor\frac{n}{2}\right\rfloor$ we get (11) by using Lemma 1. Lemma 1 and Lemma 2 give us (12).

It follows from (2) and (3) that the lower bound presented in Theorem 1 is $2^{n-o(n)}$. Our games are easily seen to be within a factor $n$ from the upper bound from (1). Finally, we point out that the proof of the lower bound in [6] is constructive.

\section{References}

[1] E. Agrell, A. Vardy, and K. Zeger. Upper bounds for constant-weight codes. IEEE Transactions on Information Theory, 46:2373-2395, 2000.

[2] W.-S. Cheung and T.-W. Ng. A three-dimensional voting system in Hong Kong. European Journal of Operational Research, 236(1):292-297, 2014.

[3] E. Elkind, L. A. Goldberg, P. W. Goldberg, and M. Wooldridge. On the dimensionality of voting games. In Proc. 23rd AAAI Conference on Artificial Intelligence, pages 69-74. AAAI Press, 2008.

[4] J. Freixas. The dimension for the European Union Council under the Nice rules. European Journal of Operational Research, 156(2):415-419, 2004.

[5] J. Freixas and M.A. Puente. A note about games-composition dimension. Discrete Applied Mathematics, 113(23):265-273, 2001.

[6] R.L. Graham and N.J.A. Sloane. Lower bounds for constant weight codes. IEEE Transactions on Information Theory, 26(1):37-43, 1980. 
[7] D.M. Kilgour. A formal analysis of the amending formula of canada's constitution act, 1982. Canadian Journal of Political Science/Revue Canadienne de Science Politique, 16:771-777, 1983.

[8] S. Kurz and S. Napel. Dimension of the Lisbon voting rules in the EU council: a challenge and new world record. Optimization Letters, pages 1-12, to appear. doi: 10.1007/s11590-015-0917-0.

[9] D. Lubell. A short proof of Sperner's lemma. Journal of Combinatorial Theory, 1(2):299-299, 1966.

[10] Y.L. Luke. The special functions and their approximations. Academic Press New York, 1969.

[11] A. Taylor and W. Zwicker. Weighted voting, multicameral representation, and power. Games and Economic Behavior, 5(1):170-181, 1993.

[12] A.D. Taylor and W.S. Zwicker. Simple games: desirability relations, trading, and pseudoweightings. Princeton University Press, New Jersey, USA, 1999.

[13] S.B. Wicker. Error Control Systems for Digital Communication and Storage. Prentice-Hall, Inc., Upper Saddle River, NJ, USA, 1995. 